\title{
Pharmacogenomics of anti-platelet therapy: how much evidence is enough for clinical implementation?
}

\author{
Christina G Perry ${ }^{1}$ and Alan R Shuldiner ${ }^{1,2}$ \\ Pharmacogenomics, the study of the genomics of drug response and adverse effects, holds great promise for more effective \\ individualized (personalized) medicine. Recent evidence supports a role of loss-of-function (LOF) variants in the cytochrome P450 \\ enzyme CYP2C19 as a determinant of clopidogrel response. Patients given clopidogrel after percutaneous coronary intervention \\ who carry LOF variants do not metabolize clopidogrel, a prodrug, into its active form resulting in decreased inhibition of platelet \\ function and a higher likelihood of recurrent cardiovascular events. Despite a large body of evidence supporting clinical utility, \\ adoption of anti-platelet pharmacogenetics into clinical practice has been slow. In this review, we summarize the pharmacokinetic, \\ pharmacodynamic and clinical evidence, identify gaps in knowledge and other barriers that appear to be slowing adoption, and \\ describe CYP2C19 pharmacogenetics implementation projects currently underway. Only when we surmount these barriers will \\ clinicians be able to use pharmacogenetic information in conjunction with the history, physical examination and other medical \\ tests and information to choose the most efficacious anti-platelet therapy for each individual patient.
}

Journal of Human Genetics (2013) 58, 339-345; doi:10.1038/jhg.2013.41; published online 23 May 2013

Keywords: anti-platelet therapy; clopidogrel; coronary artery disease; percutaneous coronary intervention; personalized medicine; pharmacogenomics; platelet function; Plavix

\section{INTRODUCTION}

Anti-platelet medications are a widely prescribed drug class used in the primary and secondary prevention of thrombotic events associated with cardiovascular disease. The adenosine diphosphate (ADP) receptor inhibitors are a subclass of anti-platelet medications, which include clopidogrel, prasugrel, ticagrelor and ticlopidine. Clopidogrel is currently one of the most commonly prescribed medications in the United States and world-wide, indicated in patients with acute coronary syndrome (ACS) and in patients undergoing percutaneous coronary intervention (PCI). ${ }^{1,2}$ Although effective in most patients, clopidogrel exhibits large inter-individual variability, which has prompted a large amount of research to better characterize its absorption, metabolism, excretion and action toward a better understanding of the mechanisms underlying its variable response. Recent findings show that common genetic variation can explain an appreciable portion of inter-individual variation in clopidogrel response. With the addition of new anti-platelet therapy alternatives for those with a genetic predisposition toward inadequate response, the promise of translating these pharmacogenetic insights into more effective individualized anti-platelet therapy has sparked much excitement and optimism for the future of personalized medicine.

\section{CLOPIDOGREL (PLAVIX)}

Clopidogrel is an oral thienopyridine prodrug often prescribed in combination with aspirin. Upon ingestion, absorption from the gastrointestinal tract into the bloodstream is regulated by ATP-binding cassette, sub-family B, member 1 (ABCB1) transporters located on the cell surface of intestinal epithelial cells, which transport clopidogrel back into the intestinal lumen inhibiting absorption.,4 Once absorbed, $\sim 85 \%$ of clopidogrel is hydrolyzed in the liver during first-pass metabolism by esterases, primarily carboxylesterase 1 (CES1), to create inactive metabolites. ${ }^{5}$ Conversion of the remaining $\sim 15 \%$ of clopidogrel from prodrug to active metabolite also occurs in the liver by two sequential oxidation steps that involve several cytochrome P450 (CYP450) enzymes (for example, CYP1A2, CYP2B6, CYP2C9, CYP2C19 and CYP2A4/5). ${ }^{6}$ Upon activation, clopidogrel acts to decrease platelet aggregation by selectively and irreversibly inhibiting the $\mathrm{P}_{2} \mathrm{Y}_{12}$ receptor located on the surface of platelets. The inability of ADP to activate $P 2 Y_{12}$ receptors prevents the associated $G_{i}$ proteins from inhibiting adenylyl cyclase, resulting in an increase in expression of cyclic adenosine monophosphate (cAMP), which prevents the activation of phosphoinositide 3-kinase (PI3K), and subsequently decreases expression of glycoprotein IIb/IIIa. ${ }^{7}$ The expression of glycoprotein IIb/IIIa on the surface of platelets mediates fibrinogen binding, leading to platelet aggregation, endothelial adherence and thrombus formation. Thus, prevention of this pathway by clopidogrel and other ADP receptor inhibitors results in decreased platelet aggregation.

The inter-individual variability of platelet inhibitory response by clopidogrel is well established. ${ }^{8-12}$ Clinical predictors of response

1Program in Personalized and Genomic Medicine, and the Division of Endocrinology, Diabetes and Nutrition, Department of Medicine, University of Maryland School of Medicine, Baltimore, MD, USA and ${ }^{2}$ Geriatric Research and Education Clinical Center, Veterans Administration Medical Center, Baltimore, MD, USA

Correspondence: Professor AR Shuldiner, University of Maryland School of Medicine, 685 West Baltimore Street, Room 379, Baltimore, MD 21201, USA.

E-mail: ashuldin@medicine.umaryland.edu

Received 4 April 2013; accepted 11 April 2013; published online 23 May 2013 
include high pre-treatment platelet reactivity, use of proton pump inhibitors, lipophilic statins, calcium channel blockers, or St John's Wort, and smoking - all of which are associated with a decreased clopidogrel response. ${ }^{8,13-16}$ Combined, these factors only account for a small fraction of the variation of response to clopidogrel while the majority of variability remains unexplained. Clinically, increased ontreatment platelet aggregation is associated with a greater frequency of recurrent thrombotic events whereas low on-treatment platelet aggregation is associated with increased bleeding risk. ${ }^{17-24}$ Platelet function testing may be used to identify patients with high ontreatment platelet aggregation; however, for logistical and other reasons, use of platelet function testing in clinical care remains controversial. ${ }^{18,25-27}$

\section{CYTOCHROME P450, FAMILY 2 C, POLYPEPTIDE 19 (CYP2C19) AND CLOPIDOGREL RESPONSE}

As described above, hepatic biotransformation of clopidogrel prodrug to its active metabolite requires CYP450 enzymes, particularly CYP2C19. In addition to clopidogrel, the CYP2C19 enzyme takes part in the metabolism of numerous drugs used in clinical practice such as antidepressants, benzodiazepines, mephenytoin and some proton pump inhibitors. ${ }^{6}$ The gene CYP2C19, which encodes the CYP2C19 enzyme, has over 25 known variant alleles with the wildtype allele, CYP2C19*1, being associated with normal CYP2C19 function. ${ }^{28,29}$ Common loss-of-function (LOF) variants in CYP2C19 are the most well-established genetic determinants of clopidogrel responsiveness. ${ }^{4}$ The most common CYP2C19 LOF allele is ${ }^{*} 2$ (c.681G $>A$; rs4244285) with allele frequencies of $\sim 15 \%$ in Caucasians and Africans, and 29-35\% in Asians. Additional CYP2C19 reduced-function alleles have been identified (for example, ${ }^{*} 3-{ }^{*} 8$ ), all of which have allele frequencies below $1 \%$, with the exception of $*_{3}$ (c.636G > A; rs4986893) with an allele frequency of 2-9\% in Asians. At the molecular level, ${ }^{\star} 2$ and ${ }^{\star} 7$ alleles cause a splicing defect, the ${ }^{*} 3$ allele results in the addition of a premature stop codon, the $\star^{\star} 4$ allele causes a mutation in the initiation codon, and ${ }^{*} 5$ and ${ }^{*} 6$ alleles contain deleterious missense mutations, ${ }^{30,31}$ each resulting in an inactive CYP2C19 protein product. Conversely, CYP2C19*17 (c.-806C > T; rs12248560) is associated with increased CYP2C19 transcription, resulting in a modest gain of function. The ${ }^{*} 17$ allele is relatively common with an average multi-ethnic allele frequency of $\sim 3-21 \%$. Owing to linkage disquilibrium, the ${ }^{\star} 17$ allele is carried almost exclusively on the common ${ }^{*} 1$ haplotype, that is, an allele containing both ${ }^{*} 2$ and ${ }^{*} 17$ variants is very rare or non-existent. ${ }^{30,32}$

CYP2C19 alleles are inherited as autosomal co-dominant traits allowing individuals to be assigned to likely CYP2C19 metabolizer phenotypes based on their CYP2C19 genotype. The metabolizer phenotypes and associated genotypes include ultra-rapid metabolizers (UMs; ${ }^{*} 1 /^{\star} 17,{ }^{*} 17 /{ }^{*} 17$ ), extensive metabolizers (EMs; ${ }^{*} 1 /{ }^{*} 1$ ), intermediate metabolizers (IMs; ${ }^{*} 1 /^{*} 2,{ }^{*} 1 /^{*} 3$ ) and poor metabolizers $\left(\mathrm{PMs} ;{ }^{*} 2 /^{\star} 2,{ }^{*} 2{ }^{*} 3,{ }^{*} 3 /^{*} 3\right) .6$ A small percentage of individuals will carry one LOF and one ${ }^{\star} 17$ allele, which are usually classified as IMs. The frequency of IMs and PMs is $\sim 18-45 \%$ and $\sim 2-15 \%$, respectively, with populations of Caucasian and African background at the lower end of these ranges and populations of Asian background at the higher end of these ranges. Based on knowledge of CYP2C19 metabolizer phenotype and its role in the activation of clopidogrel, one would predict ultra-rapid metabolizers and extensive metabolizers to have adequate concentrations of the active metabolite for effective platelet inhibition and cardioprotection, while PMs would be expected to have decreased concentrations of active metabolite, increased on-treatment platelet aggregation and inadequate cardioprotection, with IMs response to clopidogrel falling somewhere in between.

\section{EVIDENCE FOR ASSOCIATION OF CYP2C19 GENOTYPE WITH CLOPIDOGREL PHARMACOKINETICS, PHARMACODYNAMICS AND CLINICAL OUTCOMES}

Early evidence for a role of pharmacogenetics in clopidogrel response came from small pharmacokinetic and pharmacodynamics studies. ${ }^{33-37}$ In an effort to determine if functional variants in genes coding for candidate CYP450 enzymes influence platelet responsiveness to clopidogrel, Hulot et al. ${ }^{33}$ performed a prospective pharmacogenetic study consisting of 28 healthy white male volunteers treated for 7 days with $75 \mathrm{mg}$ per day of clopidogrel. Of the four CYP450 variant alleles tested, only the CYP2C19² LOF allele was significantly associated with a marked decrease in platelet responsiveness to clopidogrel compared with ${ }^{*} 1 /{ }^{*} 1$ homozygotes; no association was found between CYP2C19 genotype and baseline platelet activity, consistent with a true pharmacogenetic effect. Umemura et al. ${ }^{34}$ performed a similar study using 47 healthy Asian subjects to demonstrate that the CYP2C19 genotype status is a determining factor for the formation of the active metabolite of clopidogrel.

A number of subsequent studies support a relationship between CYP2C19 genotype and clopidogrel active metabolite levels and residual platelet reactivity. ${ }^{38-40}$ In the Pharmacogenomics of Anti-Platelet Intervention (PAPI) Study, 420 healthy Amish subjects, all of whom are related through a complex 14-generation pedigree, were exposed to clopidogrel for 1 week with ADP-stimulated platelet aggregation measured before and after intervention. ${ }^{41}$ As previously observed, there was large inter-individual variation in response (Figure 1). The estimated heritability of clopidogrel response as measured by residual ADP-stimulated platelet aggregation was 70\%. A genome-wide association study (GWAS) identified the CYP2C19 locus, specifically $C Y P 2 C 19^{*} 2$ as a major determinant of postclopidogrel ADP-stimulated platelet aggregation, explaining $\sim 12 \%$ of the variation in response in healthy individuals.

Indications that associations of CYP2C19 LOF alleles with decreased active metabolite and increased residual on-treatment platelet reactivity translated to poorer clinical outcomes came from large prospective clinical trials of clopidogrel in which stored samples were genotyped retrospectively, such as the Therapeutic Outcomes by Optimizing Platelet Inhibition with Prasugrel-Thrombolysis in Myocardial Infarction (TRITON-TIMI) 38 trial, $^{3}$ a large French acute myocardial infarction cohort $^{42}$ and others. ${ }^{41,43-45}$ Mega et al. ${ }^{46}$ conducted a meta-analysis of 9685 patients from nine previously published studies on the relationship between CYP2C19 genotype and clinical outcomes. The two end points that were examined were a composite end point comprising cardiovascular death, myocardial infarction or stroke, and stent thrombosis, a rare but potentially fatal complication of PCI. Of the 9685 patients, $54.5 \%$ had ACS and 91.3\% had undergone PCI. The results showed a significantly increased risk of the composite end point in both carriers of one (heterozygotes; HR 1.55, 95\% CI 1.11-2.17, $P=0.01$ ) and two (homozygotes; HR 1.76, 95\% CI 1.24-2.50, $P=0,002$ ) LOF CYP2C19 alleles as compared with ${ }^{*} 1$ homozygotes. Similarly, there was a significantly increased risk of stent thrombosis in both carriers of one (heterozygotes; HR 2.67, 95\% CI 1.69-4.22, $P<0.001$ ) and two (homozygotes; HR 3.97, 95\% CI 1.75-9.02, $P=0.001$ ) LOF CYP2C19 alleles compared with ${ }^{*} 1$ homozygotes. The authors concluded that, 'among patients treated with clopidogrel for percutaneous coronary intervention, carriage of even 1 reduced-function 

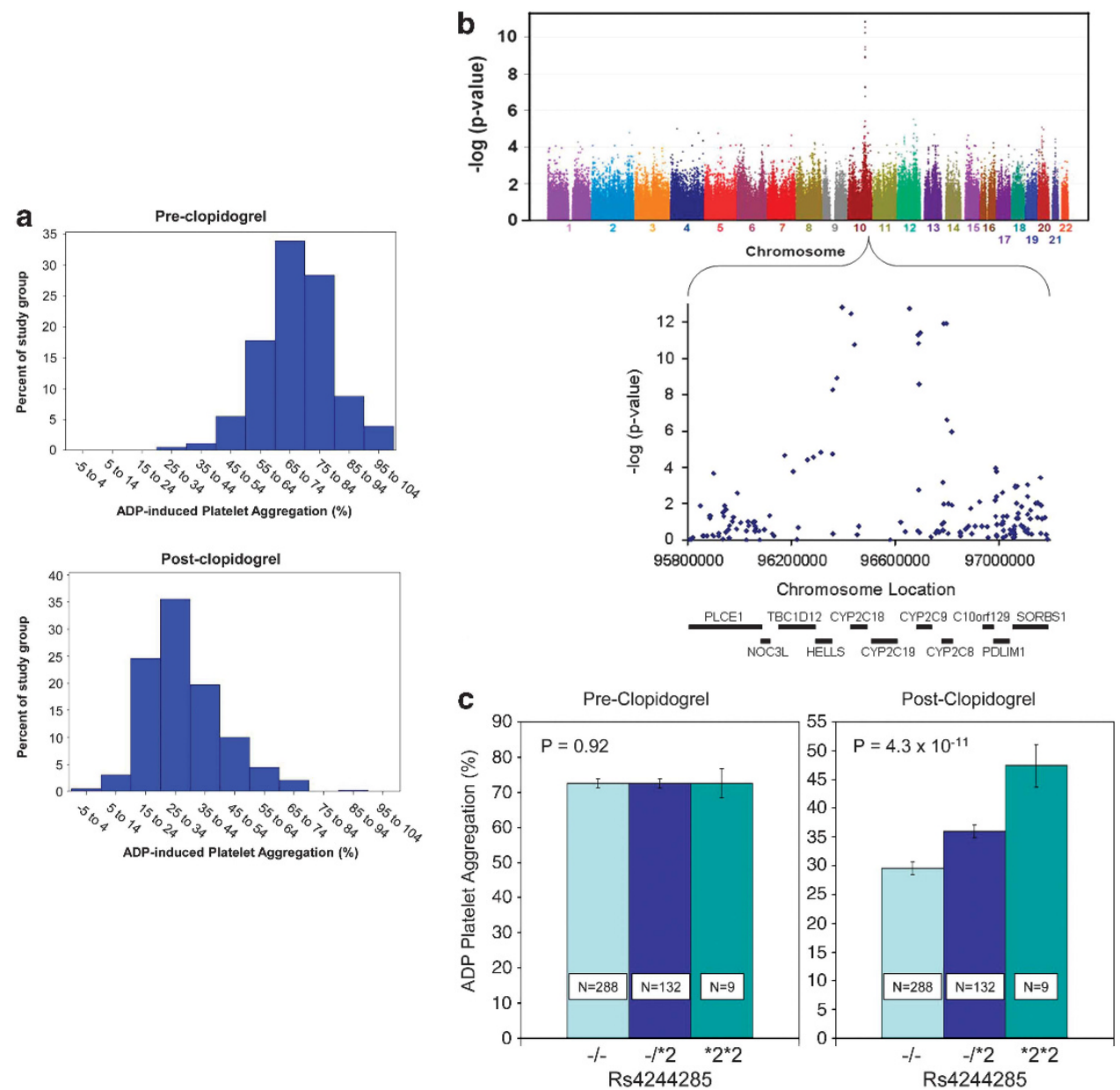

Figure 1 Summary of results from the Pharmacogenomics of Anti-platelet Intervention (PAPI) Study. (a) Distribution of ADP-stimulated platelet aggregation (\% of maximal aggregation) before (upper panel) and after (lower panel) 7 days of clopidogrel exposure in 420 PAPI Study participants. (b) Genome-wide association analysis of clopidogrel response in the PAPI Study (upper panel) revealing genome-wide significant associations in the region of the CYP2C18CYP2C19-CYP2C9-CYP2C8 cluster on chromosome 10 (lower panel). (c) Association of the loss-of-function CYP2C19*2 variant (rs4244285) was significant with ADP-stimulated platelet aggregation post-clopidogrel (right panel) but not pre-clopidogrel (left panel) in PAPI Study participants. Figure adapted from Shuldiner et al. ${ }^{41}$

CYP2C19 allele appears to be associated with a significantly increased risk of major adverse cardiovascular events, particularly stent thrombosis. $^{46}$

The increasing consistent evidence linking CYP2C19 LOF alleles to decreased clopidogrel active metabolite, increased residual on-treatment platelet reactivity, and poorer clinical outcomes provoked the U.S. Food and Drug Administration (FDA) in March 2010 to add a boxed warning to the Plavix (clopidogrel) label to alert health-care professionals and patients to the potential decreased efficacy of the drug in CYP2C19 poor metabolizers (that is, individuals carrying two LOF CYP2C19 alleles). The purpose of the FDA adding the boxed warning was 'to highlight this warning to make sure health-care professionals use the best information possible to treat their patients. ${ }^{37}$ The warning emphasizes the effect observed in CYP2C19 PMs, specifically in patients with ACS undergoing PCI, and suggests that clinicians consider other anti-platelet treatments in patients known to be CYP2C19 PMs. The label does not require genetic testing before starting a patient on clopidogrel therapy and thus if the patient's genotype is not known, the decision to perform genetic testing is left up to the individual clinician. Furthermore, the boxed warning is silent regarding recommendations for intermediate metabolizers (that is, individuals carrying one CYP2C19 LOF allele).

Despite overall consistency in the relationship between CYP2C19 genotype and clinical outcomes in patients with ACS/PCI, studies of CYP2C19 genotype in patients treated with clopidogrel for other indications such as atrial fibrillation ${ }^{48}$ and stable angina ${ }^{49}$ have been largely negative. In a recent meta-analysis by Holmes et al., ${ }^{50}$ which included 32 studies of 42016 patients over a range of indications for anti-platelet therapy, only marginal association between CYP2C19 genotype and composite cardiovascular outcomes was observed. The authors argued that small study bias might underlie previous positive associations. An alternative explanation is that the effect size of CYP2C19 on clopidogrel efficacy appears to parallel the effect of clopidogrel for a given indication; high in patients at high risk for recurrent cardiovascular events in the absence of effective antiplatelet therapy, for example, ACS/PCI, and low or non-existent for indications in which clopidogrel has a smaller effect on cardiovascular outcomes, for example, stable angina, atrial fibrillation and peripheral vascular disease. ${ }^{51-53}$ Thus, CYP2C19/clopidogrel is an example of indication-specific pharmacogenetics. ${ }^{54}$ 


\section{OTHER GENETIC DETERMINANTS OF CLOPIDOGREL RESPONSE}

Besides CYP2C19, additional genetic factors may contribute to clopidogrel's inter-individual variability. These variants are within genes that encode proteins involved in the absorption, metabolism, excretion or action of clopidogrel. A common genetic variant (c.3435C > T; rs1045642) in $A B C B 1$ results in overexpression of the $\mathrm{ABCB} 1$ protein and has been shown to be modestly associated with decreased clopidogrel active metabolite, ${ }^{55}$ increased on-treatment platelet reactivity ${ }^{56}$ and increased cardiovascular events. ${ }^{57}$ Using participants of the PAPI study, an uncommon CES1 variant (rs71647871) encoding a non-synonymous Gly143Gln substitution resulting in decreased catalytic function ${ }^{58}$ was shown to be associated with significantly higher active metabolite levels and more effective inhibition of ADP-simulated platelet aggregation. ${ }^{59}$ Paraoxonase 1 (PON1) is expressed in the liver and was not previously recognized to be involved in clopidogrel bioactivation until Bouman et al ${ }^{60}$ reported a significant association between a PON1 variant (c575A $>$ G; rs662) and active clopidogrel metabolite concentration, level of platelet inhibition and stent thrombosis. Controversially, subsequent studies have failed to replicate these findings resulting in a substantial amount of evidence that does not support a role for PON1 genotype in clopidogrel response. ${ }^{61-67}$ The gene $P 2 R Y 12$ encodes the $\mathrm{P} 2 \mathrm{Y}_{12}$ receptor on platelets that binds ATP leading to downstream platelet activation. Two common haplotypes exist, $\mathrm{H} 1$ and $\mathrm{H} 2$, which consists of two common linked genetic variants in the P2RY12 gene. The $\mathrm{H} 2$ allele is believed to be associated with increased expression of $\mathrm{P}_{2} \mathrm{Y}_{12}$ receptors ${ }^{68}$ and decreased clopidogrel response, ${ }^{69}$ however, inconsistent results from multiple studies have led to the conclusion that if $P 2 R Y 12$ variants have an effect on clopidogrel response, the effect is small. ${ }^{4}$

\section{MOVING ANTI-PLATELET PHARMACOGENETICS FROM DISCOVERY TO CLINICAL PRACTICE}

In 2009, the irreversible $\mathrm{P}_{2} \mathrm{Y}_{12}$ inhibitor, prasugrel, was approved by the FDA for use as an anti-platelet agent in patients with ACS. In the overall TIMI-38 Study, prasugrel was superior to clopidogrel in its ability to decrease cardiovascular events, but was also associated with increased major bleeding events. ${ }^{70}$ More recently, the reversible $\mathrm{P}_{2} \mathrm{Y}_{12}$ inhibitor, ticagrelor, was FDA approved and, similarly to prasugrel, was associated with overall superior cardiovascular outcomes when compared to clopidogrel. ${ }^{71}$ Prasugrel is a prodrug but does not require CYP2C19 for bioactivation while ticagrelor does not require bioactivation. During this same time period, clopidogrel has come off patent and thus inexpensive generic formulations are available. This collusion of events suggests a 'perfect storm' for the application of pharmacogenetics to individualized anti-platelet therapy in which CYP2C19 ultra-rapid metabolizers and extensive metabolizers might receive clopidogrel, reserving the more expensive agents (also associated with more bleeding events) for CYP2C19 IMs and PMs.

Despite a compelling body of data supporting clinical relevance of CYP2C19 genotype in clopidogrel-treated ACS/PCI patients, to date, there have been no randomized prospective clinical trials that have demonstrated that genotype-directed therapy is more effective than conventional approaches. This, combined with other factors (see below) has led to controversy with regard to the role of CYP2C19 genetic testing in patient care. Recent guidelines issued by the American College of Cardiology Foundation/American Heart Association $^{27}$ suggest a Class IIb recommendation for CYP2C19 genetic testing in patients with ACS. Class IIb indicates conditions for which usefulness/efficacy is less well established by evidence or opinion. The consensus guideline states, 'on the basis of the current evidence, it is difficult to strongly recommend [CYP2C19] genotype testing routinely in patients with ACS, but it might be considered on a case-by-case basis, especially in patients who experience recurrent ACS events despite ongoing therapy with clopidogrel.' The article goes on to state, 'The Class IIb recommendation of these strategies suggests that a selective, limited approach to platelet [CYP2C19] genotype assessment and platelet function testing is the more prudent course until better clinical evidence exists for us to provide a more scientifically derived recommendation. ${ }^{27}$

A major challenge to establishing the evidence base for or against implementation of CYP2C19 genetic testing, and indeed for the field of pharmacogenetics more generally, is that properly powered definitive prospective randomized clinical trials of genotype-directed therapy may not be feasible. Such clinical trials are expensive, time consuming and are unlikely to address the logistical issues necessary for effective implementation in most real-world settings. Therefore, it may be necessary to resort to pragmatic clinical trials in which early adopters develop evidence for or against clinical efficacy and cost effectiveness over time. It is in this light that the Clinical Pharmacogenetics Implementation Consortium (CPIC) provides recommendations and suggested genotype-directed treatment algorithms for drug-gene pairs for which there is reasonable evidence for clinical utility. ${ }^{6}$ CPIC guidelines suggest initiating CYP2C19 genotyping as early as possible in patients in the acute PCI setting if CYP2C19 genotype is not already known. CPIC's 2013 update also stresses that these genotyping recommendations are applicable only to patients with ACS and/or those undergoing PCI given the burden of evidence regarding increased risk of stent thrombosis in CYP2C19 IMs and PMs. As described above, the use of CYP2C19 genotyping in other non-ACS/PCI clopidogrel-treated patient populations is not supported by experimental data.

Genetic testing for $\mathrm{CYP} 2 \mathrm{C} 19{ }^{*} 2,{ }^{*} 3,{ }^{*} 17$ and other variants is currently offered by a number of commercial Clinical Laboratory Improvement Amendments (CLIA)-certified laboratories. However, the turn-around time is typically several days to more than a week, which is suboptimal in acute medical settings, particularly post PCI in which stent thrombosis is an early, and sometimes fatal, complication of ineffective anti-platelet therapy. In this situation, rapid and reliable point of care genotyping is desirable. Indeed a number of options are emerging for this purpose (for example, Spartan Bioscience RX CYP2C19; Nanosphere Verigene CYP2C19; GeneMark Dx eSensor $\mathrm{XT}-8)$.

Recently, Roberts et al. ${ }^{72}$ performed a prospective, randomized proof-of-concept trial (RAPID GENE trial) to assess the ability of a rapid turn-around point-of-care CYP2C19 genetic test to identify patients' $C Y P 2 C 19^{*} 2$ carrier status and to evaluate the possible benefits of genotype-directed personalization of anti-platelet treatment. Two hundred patients undergoing PCI for ACS or stable angina were randomly assigned to either the rapid genotyping arm of the study or the standard treatment arm, and treated with an ADP receptor inhibitor anti-platelet medication for 7 days. Patients in the rapid genotyping arm were treated with $75 \mathrm{mg}$ of clopidogrel daily if they did not carry any copies of the CYP2C19 2 allele or $10 \mathrm{mg}$ prasugrel daily if they carried one or more copies of the CYP $2 C 19^{\star} 2$ allele. All of the patients in the standard treatment arm of the study received $75 \mathrm{mg}$ clopidogrel daily and were not genotyped until after the 7 day period. Platelet function measurements were obtained on all patients on day 7 . None of the $23 C Y P 2 C 19^{*} 2$ carriers in the prasugrel-treated rapid genotyping arm had a $\mathrm{P}_{2} \mathrm{Y}_{12}$ reactivity unit (PRU) value, a measurement of on-treatment platelet reactivity, of 
more than 234 at day 7 , compared with 7 of 23 CYP2C19*2 carriers (30\%) given standard clopidogrel treatment $(P=0.0092)$. There were no significant differences in the proportion of patients above this PRU threshold between genotype-directed and standard treatment groups in patients homozygous for the wild-type CYP2C19* 1 allele. A posthoc analysis using a lower residual platelet reactivity threshold (PRU <208) was performed based upon results of the GRAVITAS trial, ${ }^{73}$ suggesting that a PRU $<208$ was associated with improved clinical outcomes. Analysis using the lower threshold yielded results similar to the primary analysis with 11 of 23 carriers (48\%) given standard treatment having PRU values $>208$ at day 7 compared with 1 of 23 carriers $(4 \%)$ in the rapid genotyping group $(P=0.0017)$. The authors' interpretations of these results were that 'point-of-care genetic testing after PCI can be done effectively at the bedside and treatment of identified $\mathrm{CYP} 2 \mathrm{C} 19^{*} 2$ carriers with prasugrel can reduce high on-treatment platelet reactivity. ${ }^{72}$ Although these results are encouraging, given that residual on-treatment platelet reactivity is associated with poorer clinical outcomes, the study was not powered, nor were patients followed long enough, to directly demonstrate improved clinical outcomes in the genotype-directed anti-platelet therapy group compared with the standard therapy group.

\section{OVERCOMING BARRIERS TO IMPLEMENTATION OF ANTI-PLATELET PHARMACOGENETICS}

A recent review by Manolio et al. ${ }^{74}$ describes the challenges to implementation of genomic medicine. These include limited evidence and conflicting interpretation of benefit/value, often due to lack of prospective randomized clinical trials; lack of institutional and clinician acceptance; limited access to genomic medicine expertise and testing; lack of standards for genomic applications; inadequate electronic health record integration of genomic results and clinical decision support, follow-up of genotyped patients, consent and understanding by clinicians, patients and the public; and lack of reimbursement by insurance companies for testing. All of these are applicable to the under-utilization of CYP2C19 genetic testing in clinical practice today, despite substantial evidence supporting clinical utility and the FDA label suggesting its use. Until these barriers to implementation are traversed, a futile cycle will persist in which clinicians remain confused about which actions to take if any, while professional associations await 'high-quality' evidence from large prospective randomized trials before creating consensus guidelines and third-party payers agree to reimbursement for testing. ${ }^{75}$

Furthermore, for CYP2C19 genetic testing to be widely adopted, it must be shown to be cost effective. Besides the cost of the test itself, the alternative medication for patients identified as IMs and PMs (for example, prasugrel, ticagrelor) are considerably more expensive than the current standard-of-care anti-platelet medication, clopidogrel. These costs must be offset by potential cost-savings from decreased cardiovascular events for genotype-directed anti-platelet therapy and/or the cost of treating all patients with the newer alternative agents. Indeed, simulation models suggest that genotypedirected anti-platelet therapy is more effective and/or less costly when compared with selection of clopidogrel or prasugrel in all patients. ${ }^{76}$ As CYP2C19 genotyping is implemented in various health-care settings, it will be important to perform real-world cost and effectiveness analyses.

Another barrier to clinical implementation of CYP2C19 pharmacogenetics is the return of genetic results into a patient's electronic health record, which currently has no standard format. For time and cost purposes, this is worth addressing to prevent repeat pharmacogenetic testing as well as increasing the quality of patient care.
In addition, inexperience of many clinicians to interpret and act upon genetic information, a lack of clear recommendations for pharmacogenetic testing by professional associations, and a non-existing information structure to provide decision support for genomic medicine all add to the complexity of clinicians utilizing pharmacogenetic testing as a tool to manage patients. ${ }^{77}$ In contrast to these barriers, a factor that favors adoption of pharmacogenetics into clinical care is that ethical, legal and social issues surrounding use of genomics to direct therapy seem less daunting than use of genomics for prediction of disease risk, as the pharmacogenetic test has health implications only when the drug is prescribed.

The Translational Pharmacogenomics Program of the National Institutes of Health Pharmacogenomics Research Network is a coordinated effort to identify and overcome the aforementioned barriers to implementation of evidence-based pharmacogenetics into real-world clinical practice. ${ }^{77}$ In this context, the University of Maryland Medical Center is among the early adopters of rapid turnaround CYP2C19 testing, offering PCI patients testing for CYP2C19*2, ${ }^{*} 3$ and ${ }^{*} 17$ with a 4 -h turn-around time. This information, along with clinical and other information, is being used by the treating physician to optimize anti-platelet therapy for each patient.

With CYP2C19 genotyping being relatively inexpensive and straightforward, another model for implementation into patient care is pre-emptive genotyping. With this approach, patients predicted to have a high likelihood of requiring anti-platelet therapy in the future are genotyped pre-emptively. When coupled with an electronic health record and clinical decision support, CYP2C19 genotype information can be immediately available to guide anti-platelet therapy at the time it is indicated. The Pharmacogenomic Resource for Enhanced Decisions in Care and Treatment (PREDICT) Project initiated at the Vanderbilt University Medical Center is one example of preemptive pharmacogenetics in which more than 10000 patients have been genotyped on a multiplex platform that include CYP2C19 variants. $^{78}$ The genotype data is deposited in the electronic health record for point-of-care decision support at the time clopidogrel is ordered. In the future, the pre-emptive pharmacogenetic model may be further enabled by the increasing number of direct-to-consumer genetic testing and DNA sequencing services. Thus, the number of patients who know their CYP2C19 genotype before requiring antiplatelet treatment may increase in the coming years.

\section{CONCLUSIONS}

There is impressive and consistent evidence that CYP2C19 LOF variants are determinants of clopidogrel pharmacokinetics and pharmacodynamics. CYP2C19 LOF variants have been demonstrated to be clinically significant determinants of poor outcomes in ACS/PCI patients, particularly those in whom stents have been placed. It is this patient population in whom CYP2C19 genetic testing should be strongly considered.

Despite the promise of pharmacogenetics, implementation into clinical practice has been slow. Ongoing CYP2C19 implementation efforts by early adopters are in progress using a number of models. Identification of barriers to implementation of pharmacogenetics and the development of real-world solutions is an important step forward and ultimately may enable pragmatic clinical trials to advance the evidence base and to evaluate the cost and effectiveness of CYP2C19 genetic testing. Only when we surmount these barriers will clinicians be able to use this information in conjunction with the history, physical examination and other medical tests and information to 
choose the most efficacious anti-platelet therapy for each individual patient.

\section{CONFLICT OF INTEREST}

Dr. Shuldiner receives grant support from National Institutes of Health for the study of the pharmacogenomics of anti-platelet medications. He also serves as a consultant for United States Diagnostic Standards, Inc. Ms. Perry has no conflict of interest.

\section{ACKNOWLEDGEMENTS}

This work was supported by National Institutes of Health grants U01 GM074518 and U01 HL105198, the Baltimore Veterans Administration Geriatric Research and Education Clinical Center and the University of Maryland Medical Scientist Training Program (MSTP) grant.

1 Kushner, F. G., Hand, M., Smith, S. C. Jr, King, S. B. 3rd, Anderson, J. L., Antman, E. M. et al. 2009 focused updates: ACC/AHA guidelines for the management of patients with ST-elevation myocardial infarction (updating the 2004 guideline and 2007 focused update) and ACC/AHA/SCAI guidelines on percutaneous coronary intervention (updating the 2005 guideline and 2007 focused update), a report of the American College of Cardiology Foundation/American Heart Association Task Force on Practice Guidelines. J. Am. Coll. Cardiol. 54, 2205-2241 (2009).

2 Anderson, J. L., Adams, C. D., Antman, E. M., Bridges, C. R., Califf, R. M., Casey, D. E. Jr et al. ACC/AHA 2007 guidelines for the management of patients with unstable angina/non ST-elevation myocardial infarction: a report of the American College of Cardiology/American Heart Association Task Force on Practice Guidelines (Writing Committee to Revise the 2002 Guidelines for the Management of Patients With Unstable Angina/Non ST-Elevation Myocardial Infarction): developed in collaboration with the American College of Emergency Physicians, the Society for Cardiovascular Angiography and Interventions, and the Society of Thoracic Surgeons: endorsed by the American Association of Cardiovascular and Pulmonary Rehabilitation and the Society for Academic Emergency Medicine. Circulation 116, e148-e304 (2007).

3 Mega, J. L., Close, S. L., Wiviott, S. D., Shen, L., Walker, J. R., Simon, T. et al. Genetic variants in ABCB1 and CYP2C19 and cardiovascular outcomes after treatment with clopidogrel and prasugrel in the TRITON-TIMI 38 trial: a pharmacogenetic analysis. Lancet 376, 1312-1319 (2010)

4 Fisch, A. S., Perry, C. G., Stephens, S. H., Horenstein, R. B. \& Shuldiner, A. R. Pharmacogenomics of anti-platelet and anti-coagulation therapy. Curr. Cardiol. Reports; in press (2013).

5 Ancrenaz, V., Daali, Y., Fontana, P., Besson, M., Samer, C., Dayer, P. et al. Impact of genetic polymorphisms and drug-drug interactions on clopidogrel and prasugrel response variability. Curr. Drug Metab. 11, 667-677 (2010).

6 Scott, S. A., Sangkuhl, K., Stein, C. M., Hulot, J.-S., Johnson, J. A., Roden, D. M. et al. Clinical Pharmacogenetics Implementation Consortium (CPIC) guidelines for cytochrome P450-2C19 (CYP2C19) genotype and clopidogrel therapy: 2013 Update. Clin. Pharmacol. Ther.; in press (2013).

7 Cattaneo, M. The platelet P2Y(1)(2) receptor for adenosine diphosphate: congenital and drug-induced defects. Blood 117, 2102-2112 (2011).

8 Gurbel, P. A., Bliden, K. P., Hiatt, B. L. \& O'Connor, C. M. Clopidogrel for coronary stenting: response variability, drug resistance, and the effect of pretreatment platelet reactivity. Circulation 107, 2908-2913 (2003).

9 Gurbel, P. A. \& Tantry, U. S. Drug insight: clopidogrel nonresponsiveness. Nat. Clin. Pract. Cardiovasc. Med. 3, 387-395 (2006).

10 Angiolillo, D. J., Fernandez-Ortiz, A., Bernardo, E., Alfonso, F., Macaya, C., Bass, T. A et al. Variability in individual responsiveness to clopidogrel: clinical implications, management, and future perspectives. J. Am. Coll. Cardiol. 49, 1505-1516 (2007).

11 O'Donoghue, M. \& Wiviott, S. D. Clopidogrel response variability and future therapies: clopidogrel: does one size fit all? Circulation 114, e600-e606 (2006).

12 Wang, T. H., Bhatt, D. L. \& Topol, E. J. Aspirin and clopidogrel resistance: an emerging clinical entity. Eur. Heart J. 27, 647-654 (2006).

13 Gilard, M., Arnaud, B., Le Gal, G., Abgrall, J. F. \& Boschat, J. Influence of omeprazol on the antiplatelet action of clopidogrel associated to aspirin. J. Thromb. Haemost. 4 , 2508-2509 (2006)

14 Lau, W. C., Gurbel, P. A., Watkins, P. B., Neer, C. J., Hopp, A. S., Carville, D. G. et al. Contribution of hepatic cytochrome P450 3A4 metabolic activity to the phenomenon of clopidogrel resistance. Circulation 109, 166-171 (2004).

15 Siller-Matula, J. M., Lang, I., Christ, G. \& Jilma, B. Calcium-channel blockers reduce the antiplatelet effect of clopidogrel. J. Am. Coll. Cardiol. 52, 1557-1563 (2008).

16 Cayla, G., Hulot, J. S., O'Connor, S. A., Pathak, A., Scott, S. A., Gruel, Y. et al. Clinical, angiographic, and genetic factors associated with early coronary stent thrombosis. JAMA 306, 1765-1774 (2011).

17 Gurbel, P. A., Bliden, K. P., Guyer, K., Cho, P. W., Zaman, K. A., Kreutz, R. P. et al. Platelet reactivity in patients and recurrent events post-stenting: results of the PREPARE POST-STENTING Study. J. Am. Coll. Cardiol. 46, 1820-1826 (2005).
18 Gurbel, P. A., Becker, R. C., Mann, K. G., Steinhubl, S. R. \& Michelson, A. D. Platelet function monitoring in patients with coronary artery disease. J. Am. Coll. Cardiol. 50, 1822-1834 (2007)

19 Angiolillo, D. J. \& Alfonso, F. Platelet function testing and cardiovascular outcomes: steps forward in identifying the best predictive measure. Thromb. Haemost. 98, 707-709 (2007).

20 Bliden, K. P., DiChiara, J., Tantry, U. S., Bassi, A. K., Chaganti, S. K. \& Gurbel, P. A. Increased risk in patients with high platelet aggregation receiving chronic clopidogre therapy undergoing percutaneous coronary intervention: is the current antiplatelet therapy adequate? J. Am. Coll. Cardiol. 49, 657-666 (2007).

21 Buonamici, P., Marcucci, R., Migliorini, A., Gensini, G. F., Santini, A., Paniccia, R. et al. Impact of platelet reactivity after clopidogrel administration on drug-eluting stent thrombosis. J. Am. Coll. Cardiol. 49, 2312-2317 (2007).

22 Gurbel, P. A., Bliden, K. P., Zaman, K. A., Yoho, J. A., Hayes, K. M. \& Tantry, U. S Clopidogrel loading with eptifibatide to arrest the reactivity of platelets: results of the Clopidogrel Loading With Eptifibatide to Arrest the Reactivity of Platelets (CLEAR PLATELETS) study. Circulation 111, 1153-1159 (2005).

23 Gurbel, P. A., Antonino, M. J., Bliden, K. P., Dichiara, J., Suarez, T. A., Singla, A et al. Platelet reactivity to adenosine diphosphate and long-term ischemic event occurrence following percutaneous coronary intervention: a potential antiplatelet therapeutic target. Platelets 19, 595-604 (2008).

24 Matetzky, S., Shenkman, B., Guetta, V., Shechter, M., Beinart, R., Goldenberg, I. et al. Clopidogrel resistance is associated with increased risk of recurrent atherothrombotic events in patients with acute myocardial infarction. Circulation 109, 3171-3175 (2004)

25 Dahlen, J. R., Price, M. J., Parise, H. \& Gurbel, P. A. Evaluating the clinical usefulness of platelet function testing: considerations for the proper application and interpretation of performance measures. Thromb. Haemost. 109, 808-816 (2012).

26 Collet, J. P., Cuisset, T., Range, G., Cayla, G., Elhadad, S., Pouillot, C. et al. Bedside monitoring to adjust antiplatelet therapy for coronary stenting. N. Engl. J. Med. 367, 2100-2109 (2012).

27 Jneid, H. The 2012 ACCF/AHA focused update of the Unstable Angina/NonST-Elevation Myocardial Infarction (UA/NSTEMI) Guideline: a critical appraisal. Methodist. DeBakey Cardiovasc. J. 8, 26-30 (2012).

28 Scott, S. A., Sangkuhl, K., Shuldiner, A. R., Hulot, J. S., Thorn, C. F., Altman, R. B. et al. PharmGKB summary: very important pharmacogene information for cytochrome P450, family 2, subfamily C, polypeptide 19. Pharmacogenet. Genomics 22 159-165 (2012)

29 CYP2C19 allele nomenclature. http://www.cypalleles.ki.se/cyp2c19.htm/ (2013)

30 Scott, S. A., Sangkuhl, K., Gardner, E. E., Stein, C. M., Hulot, J. S., Johnson, J. A et al. Clinical Pharmacogenetics Implementation Consortium guidelines for cytochrome P450-2C19 (CYP2C19) genotype and clopidogrel therapy. Clin. Pharmacol. Ther. 90, 328-332 (2011)

31 Scott, S. A., Sangkuhl, K., Gardner, E. E., Stein, C. M., Hulot, J. S., Johnson, J. A. et al. Supplemental Table S1. Commonly Tested CYP2C19 Variant Alleles and their Effect on CYP2C19 Protein (ed. S1., ST) (Clinical Pharmacology \& Therapeutics, 2011).

32 Lewis, J., Stephens, S., Horenstein, R., O'Connell, J., Ryan, K., Peer, C. et al. The CYP2C19*17 variant is not independently associated with clopidogrel response. J. Thromb. Haemcost; in press (2013).

33 Hulot, J. S., Bura, A., Villard, E., Azizi, M., Remones, V., Goyenvalle, C et al. Cytochrome P450 2C19 loss-of-function polymorphism is a major determinant of clopidogrel responsiveness in healthy subjects. Blood 108, 2244-2247 (2006).

34 Umemura, K., Furuta, T. \& Kondo, K. The common gene variants of CYP2C19 affect pharmacokinetics and pharmacodynamics in an active metabolite of clopidogrel in healthy subjects. J. Thromb. Haemost. 6, 1439-1441 (2008),

35 Gladding, P., Webster, M., Zeng, I., Farrell, H., Stewart, J., Ruygrok, P. et al. The pharmacogenetics and pharmacodynamics of clopidogrel response: an analysis from the PRINC (Plavix Response in Coronary Intervention) trial. JACC Cardiovasc. Interv. 1 , 620-627 (2008)

36 Mega, J. L., Close, S. L., Wiviott, S. D., Shen, L., Hockett, R. D., Brandt, J. T. et al. Cytochrome p-450 polymorphisms and response to clopidogrel. N. Engl. J. Med. 360, 354-362 (2009)

37 Kim, K. A., Park, P. W., Hong, S. J. \& Park, J. Y. The effect of CYP2C19 polymorphism on the pharmacokinetics and pharmacodynamics of clopidogrel: a possible mechanism for clopidogrel resistance. Clin. Pharmacol. Ther. 84, 236-242 (2008).

38 Fontana, P., Hulot, J. S., De Moerloose, P. \& Gaussem, P. Influence of CYP2C19 and CYP3A4 gene polymorphisms on clopidogrel responsiveness in healthy subjects. J. Thromb. Haemost. 5, 2153-2155 (2007)

39 Brandt, J. T., Close, S. L., Iturria, S. J., Payne, C. D., Farid, N. A., Ernest, C. S. 2nd et al. Common polymorphisms of CYP2C19 and CYP2C9 affect the pharmacokinetic and pharmacodynamic response to clopidogrel but not prasugrel. J. Thromb. Haemost. 5, 2429-2436 (2007).

40 Simon, T., Bhatt, D. L., Bergougnan, L., Farenc, C., Pearson, K., Perrin, L. et al. Genetic polymorphisms and the impact of a higher clopidogrel dose regimen on active metabolite exposure and antiplatelet response in healthy subjects. Clin. Pharmacol. Ther. 90, 287-295 (2011).

41 Shuldiner, A. R., O'Connell, J. R. Bliden, K. P. Gandhi, A., Ryan, K., Horenstein, R. B. et al. Association of cytochrome P450 2C19 genotype with the antiplatelet effect and clinical efficacy of clopidogrel therapy. JAMA 302, 849-857 (2009).

42 Simon, T., Verstuyft, C., Mary-Krause, M., Quteineh, L., Drouet, E., Meneveau, N. et al. Genetic determinants of response to clopidogrel and cardiovascular events. N. Engl. J. Med. 360, 363-375 (2009) 
43 Trenk, D., Hochholzer, W., Fromm, M. F., Chialda, L. E., Pahl, A., Valina, C. M. et al. Cytochrome P450 2C19 681G >A polymorphism and high on-clopidogrel platelet reactivity associated with adverse 1-year clinical outcome of elective percutaneous coronary intervention with drug-eluting or bare-metal stents. J. Am. Coll. Cardiol. 51, 1925-1934 (2008).

44 Collet, J. P., Hulot, J. S., Pena, A., Villard, E., Esteve, J. B., Silvain, J. et al. Cytochrome P450 2C19 polymorphism in young patients treated with clopidogrel after myocardial infarction: a cohort study. Lancet 373, 309-317 (2009).

45 Sibbing, D., Stegherr, J., Latz, W., Koch, W., Mehilli, J., Dorrler, K. et al. Cytochrome P450 2C19 loss-of-function polymorphism and stent thrombosis following percutaneous coronary intervention. Eur. Heart J. 30, 916-922 (2009).

46 Mega, J. L., Simon, T., Collet, J. P., Anderson, J. L., Antman, E. M., Bliden, K. et al. Reduced-function CYP2C19 genotype and risk of adverse clinical outcomes among patients treated with clopidogrel predominantly for $\mathrm{PCl}$ : a meta-analysis. JAMA 304, 1821-1830 (2010)

47 FDA Announces New Boxed Warning on Plavix Alerts patients, health care professionals to potential for reduced effectiveness; Vol 2013 (2010).

48 Pare, G., Mehta, S. R., Yusuf, S., Anand, S. S., Connolly, S. J., Hirsh, J. et al. Effects of CYP2C19 genotype on outcomes of clopidogrel treatment. N. Engl. J. Med. 363, 1704-1714 (2010).

49 Bhatt, D. L., Pare, G., Eikelboom, J. W., Simonsen, K. L., Emison, E. S., Fox, K. A. et al. The relationship between CYP2C19 polymorphisms and ischaemic and bleeding outcomes in stable outpatients: the CHARISMA genetics study. Eur. Heart J. 33, 2143-2150 (2012).

50 Holmes, M. V., Perel, P., Shah, T., Hingorani, A. D. \& Casas, J. P. CYP2C19 genotype, clopidogrel metabolism, platelet function, and cardiovascular events: a systematic review and meta-analysis. JAMA 306, 2704-2714 (2011).

51 Shuldiner, A. R., Vesely, M. R. \& Fisch, A. CYP2C19 genotype and cardiovascular events. JAMA 307, 1482 (2012).

52 Mega, J. L., Topol, E. J. \& Sabatine, M. S. CYP2C19 genotype and cardiovascular events. JAMA 307, 1482-1483 (2012).

53 Siasos, G., Tousoulis, D. \& Stefanadis, C. CYP2C19 genotype and cardiovascular events. JAMA 307, 1483-1484 (2012).

54 Johnson, J. A., Roden, D. M., Lesko, L. J., Ashley, E., Klein, T. E. \& Shuldiner, A. R. Clopidogrel: a case for indication-specific pharmacogenetics. Clin. Pharmacol. Ther 91, 774-776 (2012).

55 Taubert, D., von Beckerath, N., Grimberg, G., Lazar, A., Jung, N., Goeser, T. et al. Impact of P-glycoprotein on clopidogrel absorption. Clin. Pharmacol. Ther. 80, 486-501 (2006).

56 Wang, X. D., Zhang, D. F., Liu, X. B., Lai, Y., Qi, W. G., Luo, Y. et al. Modified clopidogrel loading dose according to platelet reactivity monitoring in patients carrying ABCB1 variant alleles in patients with clopidogrel resistance. Eur. J. Intern. Med. 23, 48-53 (2012).

57 Su, J., Xu, J., Li, X., Zhang, H., Hu, J., Fang, R. et al. ABCB1 C3435T polymorphism and response to clopidogrel treatment in coronary artery disease (CAD) patients: a meta-analysis. PLoS One 7, e46366 (2012).

58 Zhu, H. J., Patrick, K. S., Yuan, H. J., Wang, J. S., Donovan, J. L., DeVane, C. L. et al. Two CES1 gene mutations lead to dysfunctional carboxylesterase 1 activity in man clinical significance and molecular basis. Am. J. Hum. Genet. 82, 1241-1248 (2008).

59 Lewis, J. P., Horenstein, R. B., Ryan, K., O'Connell, J. R., Gibson, Q., Mitchell, B. D. et al. The functional G143E variant of carboxylesterase 1 is associated with increased clopidogrel active metabolite levels and greater clopidogrel response. Pharmacogenet. Genomics 23, 1-8 (2013).

60 Bouman, H. J., Schomig, E., van Werkum, J. W., Velder, J., Hackeng, C. M., Hirschhauser, C. et al. Paraoxonase-1 is a major determinant of clopidogrel efficacy. Nat. Med. 17, 110-116 (2011).

61 Price, M. J., Murray, S. S., Angiolillo, D. J., Lillie, E., Smith, E. N., Tisch, R. L. et al. Influence of genetic polymorphisms on the effect of high- and standard-dose clopidogrel after percutaneous coronary intervention: the GIFT (Genotype
Information and Functional Testing) study. J. Am. Coll. Cardiol. 59, 1928-1937 (2012).

62 Lewis, J. P., Fisch, A. S., Ryan, K., O'Connell, J. R., Gibson, Q., Mitchell, B. D. et al. Paraoxonase 1 (PON1) gene variants are not associated with clopidogrel response. Clin. Pharmacol. Ther. 90, 568-574 (2011).

63 Hulot, J. S., Collet, J. P., Cayla, G., Silvain, J., Allanic, F., Bellemain-Appaix, A. et al. CYP2C19 but not PON1 genetic variants influence clopidogrel pharmacokinetics, pharmacodynamics, and clinical efficacy in post-myocardial infarction patients. Circ. Cardiovasc. Interv. 4, 422-428 (2011).

64 Kreutz, R. P., Nystrom, P., Kreutz, Y., Miao, J., Desta, Z., Breall, J. A. et al. Influence of paraoxonase-1 Q192R and cytochrome P450 2C19 polymorphisms on clopidogrel response. Clin. Pharmacol. 4, 13-20 (2012).

65 Simon, T., Steg, P. G., Becquemont, L., Verstuyft, C., Kotti, S., Schiele, F. et al. Effect of paraoxonase-1 polymorphism on clinical outcomes in patients treated with clopidogrel after an acute myocardial infarction. Clin. Pharmacol. Ther. 90, 561-567 (2011).

66 Lewis, J. P. \& Shuldiner, A. R. Paraoxonase 1 Q192R variant and clopidogrel efficacy: fact or fiction? Circ. Cardiovasc. Genet. 5, 153-155 (2012).

67 Sibbing, D., Koch, W., Massberg, S., Byrne, R. A., Mehilli, J., Schulz, S. et al. No association of paraoxonase-1 Q192R genotypes with platelet response to clopidogrel and risk of stent thrombosis after coronary stenting. Eur. Heart J. 32, 1605-1613 (2011).

68 Szymezak, J., Moreau, C., Loriot, M. A., Durand, E., Van Viet, H., Desnos, M. et al. High on-clopidogrel platelet reactivity: genotyping can help to optimize antiplatelet treatment. Thromb. Res. 128, 92-95 (2011).

69 Staritz, P., Kurz, K., Stoll, M., Giannitsis, E., Katus, H. A. \& Ivandic, B. T. Platelet reactivity and clopidogrel resistance are associated with the $\mathrm{H} 2$ haplotype of the P2Y12-ADP receptor gene. Int. J. Cardiol. 133, 341-345 (2009).

70 Wiviott, S. D., Braunwald, E., McCabe, C. H., Montalescot, G., Ruzyllo, W., Gottlieb, S. et al. Prasugrel versus clopidogrel in patients with acute coronary syndromes. N. Engl. J. Med. 357, 2001-2015 (2007).

71 Cannon, C. P., Harrington, R. A., James, S., Ardissino, D., Becker, R. C., Emanuelsson, $\mathrm{H}$. et al. Comparison of ticagrelor with clopidogrel in patients with a planned invasive strategy for acute coronary syndromes (PLATO): a randomised double-blind study. Lancet 375, 283-293 (2010).

72 Roberts, J. D., Wells, G. A., Le May, M. R., Labinaz, M., Glover, C., Froeschl, M. et al. Point-of-care genetic testing for personalisation of antiplatelet treatment (RAPID GENE): a prospective, randomised, proof-of-concept trial. Lancet 379, 1705-1711 (2012).

73 Price, M. J., Berger, P. B., Teirstein, P. S., Tanguay, J. F., Angiolillo, D. J., Spriggs, D. et al. Standard vs high-dose clopidogrel based on platelet function testing after percutaneous coronary intervention: the GRAVITAS randomized trial. JAMA 305, 1097-1105 (2011).

74 Manolio, T. A., Chisholm, R. L., Ozenberger, B., Roden, D. M., Williams, M. S., Wilson, R. et al. Implementing genomic medicine in the clinic: the future is here. Genet. Med. 15, 258-267 (2013).

75 Beitelshees, A. L. Personalised antiplatelet treatment: a RAPIDly moving target. Lancet 379, 1680-1682 (2012).

76 Reese, E. S., Daniel Mullins, C., Beitelshees, A. L. \& Onukwugha, E. Cost-effectiveness of cytochrome P450 2C19 genotype screening for selection of antiplatelet therapy with clopidogrel or prasugrel. Pharmacotherapy 32, 323-332 (2012).

77 Shuldiner, A. R., Relling, M. V., Peterson, J. F., Hicks, J. K., Freimuth, R. R., Sadee, W. et al. The pharmacogenomics research network translational pharmacogenetics program (TPP): overcoming challenges of real-world implementation. Clin. Pharmacol. Ther. (e-pub ahead of print 19 March 2013; doi:10.1038/clpt.2013.59).

78 Pulley, J. M., Denny, J. C., Peterson, J. F., Bernard, G. R., Vnencak-Jones, C. L., Ramirez, A. H. et al. Operational implementation of prospective genotyping for personalized medicine: the design of the Vanderbilt PREDICT project. Clin. Pharmacol. Ther. 92, 87-95 (2012). 